\title{
Generalized Maxwell Fish-Eye Lens as a Beam Splitter: A Case Study in Realizing All-Dielectric Devices From Transformation Electromagnetics
}

\author{
Qin Lei (琴雷), Robert Foster, Member, IEEE, Patrick S. Grant, and Chris Grovenor
}

\begin{abstract}
A 2-D generalized Maxwell fish-eye lens has been fabricated with all-dielectric polymer/ceramic composite materials and evaluated numerically and experimentally. The graded refractive index profile of the lens was discretized into a concentric-ring structure and functioned as a two-way beam splitter. Epoxy composites, with a broad dielectric constant range that can be precisely controlled, were used to cast the lens using a low-cost manufacturing method that can readily accommodate different geometries. The lens was tested with a nearfield microwave imaging system to demonstrate its capability of controlling waves at an operating frequency of $18 \mathrm{GHz}$. The measured results had some differences from the expected behavior, which are considered in detail and provided some insights into the practical manufacturing and measurement constraints for graded dielectric composites. Possible routes to increase the number of output beams are discussed.
\end{abstract}

Index Terms-Casting, composite dielectrics, generalized Maxwell fish-eye (GMFE) lens, gradient index (GRIN) lens, power dividing lens, surface waves.

\section{INTRODUCTION}

$\mathbf{M}$ ETAMATERIALS are artificial materials whose properties depend on the geometric arrangement of the constituents, not just their chemical and physical properties. The emergence of metamaterials has enabled many electromagnetic devices, such as invisibility cloaks [1]-[3] and novel light bending and guiding lenses [4]-[7]. Conventional metamaterials are often realized using metallic resonant structures; however, the application of resonant metamaterials suffers from the inherent limitations of high loss and narrow bandwidth [8]-[10]. An increasing effort is being made to explore nonresonant metamaterials as alternatives, among which the gradient index (GRIN) medium attracts considerable attention with the assistance of transformation optics [11], [12]. Transformation optics, or transformation

Manuscript received March 2, 2017; revised May 26, 2017; accepted June 18, 2017. This work was supported by the Engineering and Physical Sciences Research Council, U.K., under Grant EP/I034548/1. The work of Q. Lei was supported by the China Scholarship Council. (Q. Lei and $R$. Foster are co-first authors.) (Corresponding authors: Robert Foster; Patrick S. Grant.)

Q. Lei, P. S. Grant, and C. Grovenor are with the Department of Materials, University of Oxford, Oxford OX1 3PH, U.K. (e-mail: patrick.grant@materials.ox.ac.uk).

R. Foster is with the School of Electronic Engineering and Computer Science, Queen Mary University of London, London E1 4NS, U.K. (e-mail: r.n.foster@ieee.org).

Color versions of one or more of the figures in this paper are available online at http://ieeexplore.ieee.org.

Digital Object Identifier 10.1109/TMTT.2017.2727495 electromagnetics (TrE), provides a powerful tool to solve the "inverse problem" of specifying material requirements to produce a given electromagnetic response, with and without materials being present in the design space. For example, it can be used to transform a traditional isotropic dielectric lens into a GRIN lens, so that the functioning of the lens relies on the varying dielectric properties throughout the lens, instead of the geometry [13].

While TrE solves one inverse problem, a second results: how to design metamaterials and materials to exhibit the required electromagnetic response. The ability to reliably customise permittivity over a broad range of values is important for realizing TrE designs, such as compressing lenses into more compact structures; this poses an enormous challenge to materials engineering. Metamaterials, despite the narrow bandwidth usually implied, have an advantage in this respect that a "unit cell" of an (infinite) array can be specified and simulated efficiently, with the geometry varied to determine the resulting change in the electromagnetic properties. Such an approach can be used to some extent to control the effective permittivity of geometrical structures using multiple dielectrics (e.g., composed of dielectrics and air, such as geometries used in 3-D printing), but has limited applicability to the design of traditional composite materials. Examples of successful implementations of GRIN lenses include drilling subwavelength holes in dielectric substrates [14] and using polymermatrix all-dielectric composites [15]. These implementations are, however, commonly restricted by limited refractive index values and uncertainties in the ability to meet target dielectric constant values for composites [16].

In addition to bends and polarization splitters [4]-[7], TrE offers new possibilities in the design of power dividers (beam splitters). Such devices are of increasing interest for the realization of photonic circuits, but TrE beam splitters are also of interest for microwave applications. An early example of a TrE-based beam splitter was reported in 2008 using embedded coordinate transformations [17]. This relied on a full $\mathrm{TrE}$ design (i.e., anisotropic permittivity and permeability) to provide "reflectionless two-way beam division," with both input and output planes flat. Although simulation only, this showed both the possibilities in designing such devices using $\operatorname{TrE}$ and also some interesting features, such as performance under oblique incidence at the input and the effect of diffraction (resulting in some "leakage" of power into the region between the beams). More recently, embedded 
coordinate transformations have been used to design 2-D and 3-D multiway (larger than two) power dividers, with both equal and unequal power division described [18]. The study was again simulation based and reliant on anisotropic permittivities and permeabilities. In contrast, the work reported here is on an all-dielectric isotropic beam splitter based on a generalized Maxwell fish-eye (GMFE) lens, with a refractive index ranging from 1.5 to 4.1 . This paper also demonstrates the possibility of achieving reliable permittivity values in composites using a simple, low-cost casting method that can readily accommodate different geometries. A similar lens has been described [19], differing from this paper in the bed-of-nails implementation method for a bulk (3-D) lens and the additional transformations used to flatten one side of the lens and control the maximum refractive index required (the reported lens was also simulation only). In contrast, the emphasis in this paper is on the use of epoxy composites with controlled permittivity and a casting method to fabricate a surface wave beam splitter. Although no transformation was used in this paper, it is clear that the design and fabrication process can readily accommodate their use. We emphasize that the sequential casting method used is not restricted to any particular geometry, and can include both bulk and surface wave devices.

The control of surface waves using a discrete set of dielectrics of controlled permittivity (as described below) forms part of, and complements, a body of work conducted by the authors and their colleagues in recent years (part of a wider interest in the research community at large). On the materials side, a number of dielectric composites [20]-[22], ferrite composites [23]-[25], and superconducting composites [26] have been developed, providing a range of permittivity and permeability values with controlled loss. The processing of these materials to understand their use in fused deposition $3-D$ printing has been a key objective [21], [22], [25], due to the fine control of spatial variation inherent to $3-\mathrm{D}$ printing. In this paper, we adopt a cost-effective casting route by mixing an epoxy matrix and barium titanate fillers together to provide a relatively wide permittivity range, complementing the existing work with a different polymer (epoxy) and the use of an alternative, proven device fabrication technology. Casting can be scaled to high volumes and at a relatively low cost; 3-D printing still suffers, in general, from slow print speeds and low volumes.

For the control of surface waves, the authors and colleagues have demonstrated the use of metasurfaces as a Luneberg lens [27] and as an all-angle absorber [28], described how to avoid the presence of singular values in permittivity profiles by modifying the surface geometry [29], and investigated surface wave cloaks and illusion devices [30], [31], including the use of a (continuously) varying substrate height to control the effective permittivity for a homogeneous substrate permittivity [32]. This paper adds to these investigations by demonstrating a new functionality (beam splitting) using a discrete set of isotropic permittivities for a fixed height. We also note that bulk lenses using a discrete set of isotropic dielectrics have also been investigated and fabricated via casting (see [33] and [34]). Here, we apply this approach for surface waves.
This paper is organized as follows. After describing the design of the beam splitting lens in Section II, the production of the necessary composite materials and fabrication of the lens are described in Section III. Experimental results are given in Section IV, with some further analysis and discussion. Some conclusions are drawn in Section V. Additional material, concerning broadband operation and other advanced issues, is contained in two appendixes.

\section{Design of the BeAm-Splitting Lens}

The MFE lens is a rotationally symmetric spherical graded index lens that performs the operation of stereographic projection, first described in [35], and used in camera lenses and for peep-holes, to give a wider field of view (at the cost of image curvature). It is one of a family of profiles that is a solution to a formula described in [36] that allows a spherically symmetric graded index lens to "form perfect geometrical images of two given concentric spheres onto each other" [37]; other solutions include the classic Luneburg and Eaton lenses and generalized forms of the Luneburg and MFE lenses [9], [38]. (The reference to "perfect geometrical images" is understood in the sense of diffraction-limited absolute instruments in ray optics; the MFE lens was the subject of some debate in recent years over whether it also performed perfect, or subwavelength, imaging in wave optics. This is not discussed in this paper; the interested reader should see [39]-[43], and references therein, for more information.) The MFE lens has been considered for use as a 0-dB coupler (crossover) [44], [45], as has a more general TrE approach [46], while the half-MFE lens has been investigated for matching waveguides of different dimensions [47] and for antennas [48]-[53].

The GMFE equation [9] is

$$
n(r)=\frac{2}{r^{1-m}+r^{1+m}}
$$

where $r$ is the (usually normalized) radius, $n(r)$ is the radial refractive index profile, and $m$ is a variable parameter, which equals one for the MFE lens. Varying $m$ from unity results in the appearance of two focal points for one source position (rather than one focal point for the MFE lens), symmetric about the straight line connecting the source, the lens center and the lens boundary opposite the source. Examples of the relative dielectric permittivity $\left(\varepsilon_{r}(r)=n^{2}(r)\right.$ for nonmagnetic materials) profile for different $m$ values are given in Fig. 1, with the corresponding ray diagrams and 2-D full wave simulation results (produced with COMSOL) shown in Fig. 2.

It is evident from Fig. 2 that reducing the value of $m$ below unity increases the angular separation of the two resulting focal points. From Fig. 1, it is apparent that the profiles all become similar as the (normalized) radius increases, irrespective of $m$, with the difference becoming increasingly negligible above approximately $r=0.7$. Decreasing $m$ has the effect of increasing the required relative dielectric permittivity, becoming increasingly important as $r \rightarrow 0$. This obviously has implications for fabrication, both in terms of producing the required materials and also in terms of the discretization process. For example, if the maximum available relative 


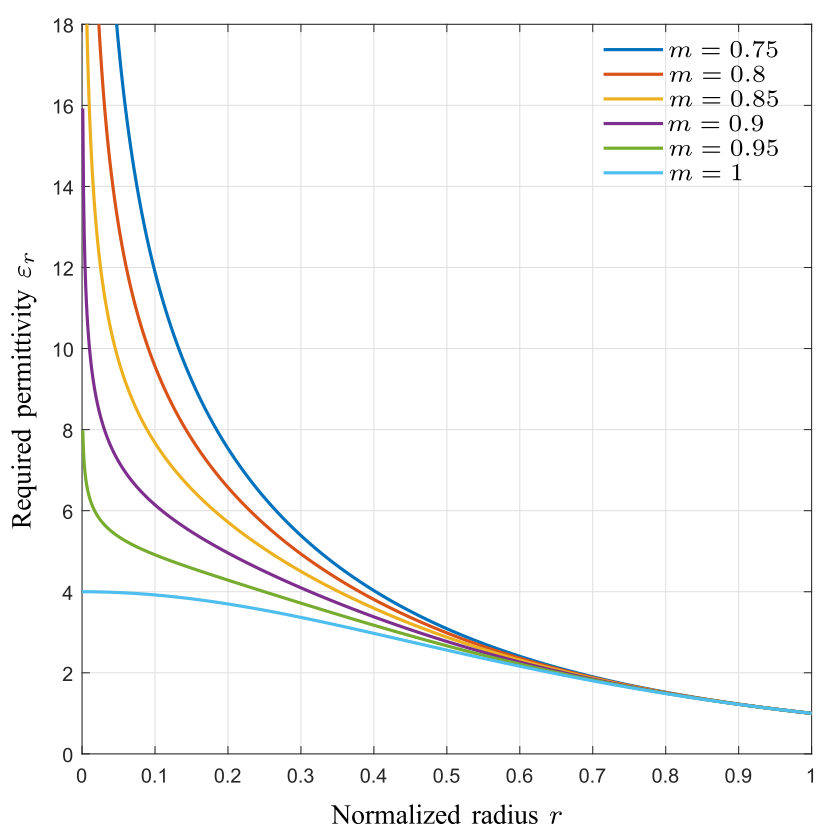

Fig. 1. Relative dielectric permittivity profiles as a function of normalized radius for the GMFE lens, for varying $m$, and including the profile for the MFE lens, when $m=1$. Note that the profiles are all singular at $r=0$ if $m \neq 1$.

dielectric permittivity was 14 , the radius of the central section would increase with decreasing $m$. This could introduce errors in the focusing capability of the GMFE lens, introducing a limit to the angular separation achievable. On the other hand, the tolerances involved in the fabrication process could be a limiting factor in this regard, depending on the size of the lens, frequency of operation, and discretized relative dielectric permittivity requirements. It is also noteworthy that the angular separation of the two focal points (a function of $m$ ) is evidently most affected by the central region of the lens; this will be relevant in Section IV.

Bulk lenses follow these profiles as given (assuming operation in air), but surface waves on a grounded dielectric see an effective refractive index (permittivity), due to the propagation through the bilayer media (air and substrate). Furthermore, surface wave devices are normally embedded in a homogeneous substrate (background substrate). Hence, a three-step process must be followed to obtain the equivalent curve for the required substrate permittivity:

1) determine the relationship between substrate permittivity and effective permittivity for a range of substrate thicknesses, permittivities, and the frequencies of interest;

2) scale the effective permittivity for the GMFE lens (for the desired value of $m$ ), obtained by squaring (1), by the effective permittivity of the background substrate;

3) use the scaled effective permittivity curve to extract the required discrete set substrate permittivities from the design curves, given a design thickness and operating frequency and material fabrication constraints.

The first step is addressed by solving [54, p. $147 \mathrm{ff}$.]

$$
k_{z_{1}} \tan \left(k_{z_{1}} d\right)-\gamma_{z_{2}} \varepsilon_{r}=0
$$

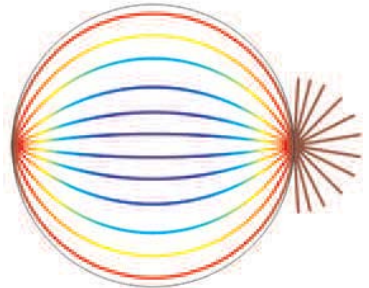

$m=1$

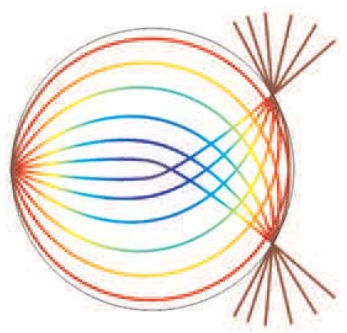

$m=0.85$

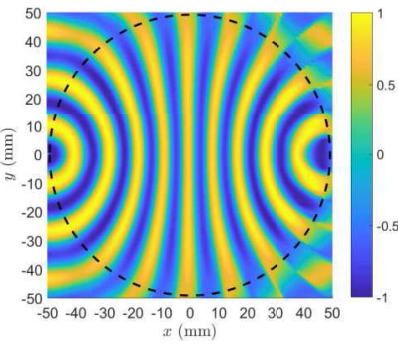

$m=1$

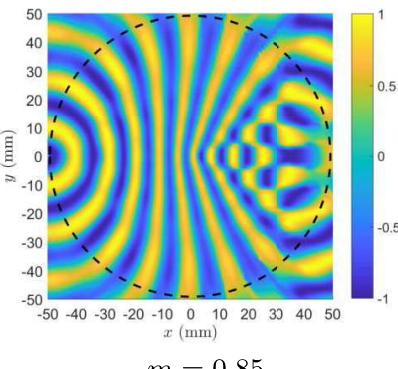

$m=0.85$

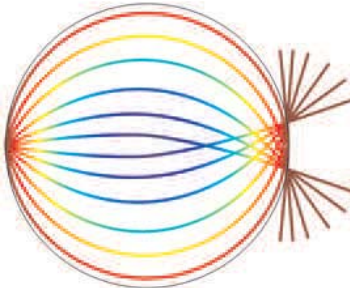

$m=0.95$

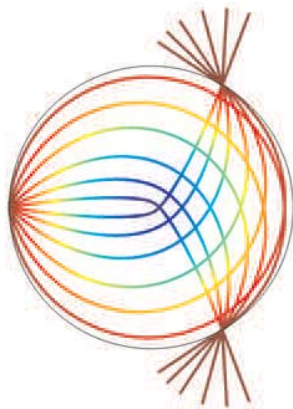

$m=0.75$

(a)

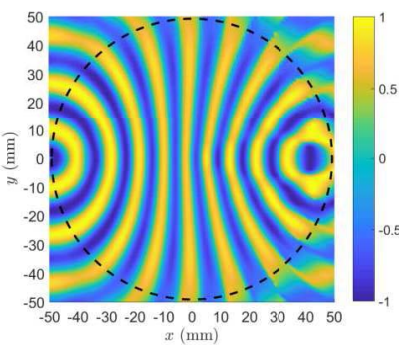

$m=0.95$

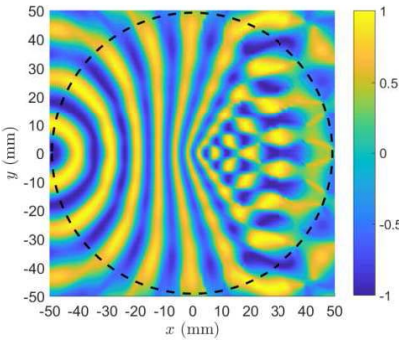

$m=0.75$

(b)

Fig. 2. (a) Ray diagrams. (b) 2-D full-wave simulations for the GMFE lens, for varying $m$, using COMSOL. (Dashed circle shows lens boundary for instantaneous $E_{z}$ field plots.)

where

$$
\begin{aligned}
& k_{z_{1}}=\sqrt{k_{0}^{2} \varepsilon_{r}-k_{x}^{2}}=k_{0} \sqrt{\varepsilon_{r}-\varepsilon_{r e}} \\
& \gamma_{z_{2}}=\sqrt{k_{x}^{2}-k_{0}^{2}}=k_{0} \sqrt{\varepsilon_{r e}-1}
\end{aligned}
$$

for the fundamental $\mathrm{TM}_{0}$ mode of interest (which has no cutoff frequency; the operating band in this paper is such that no other mode should propagate). Here, it is assumed that the wave propagates along the dielectric interface in the $x$ direction, with the $z$-axis normal to the dielectric. Region 1 is the dielectric substrate; region 2 is the air above the substrate. The wavevector components are $k_{a_{i}}$, where $a$ denotes the component direction and $i$ the region 


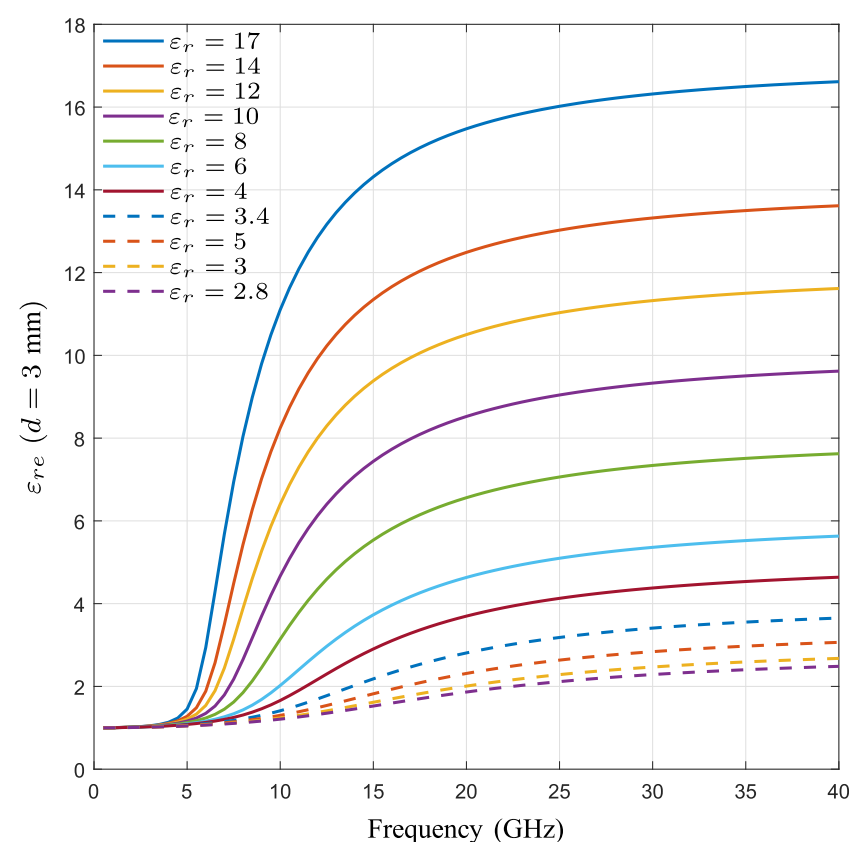

(a)

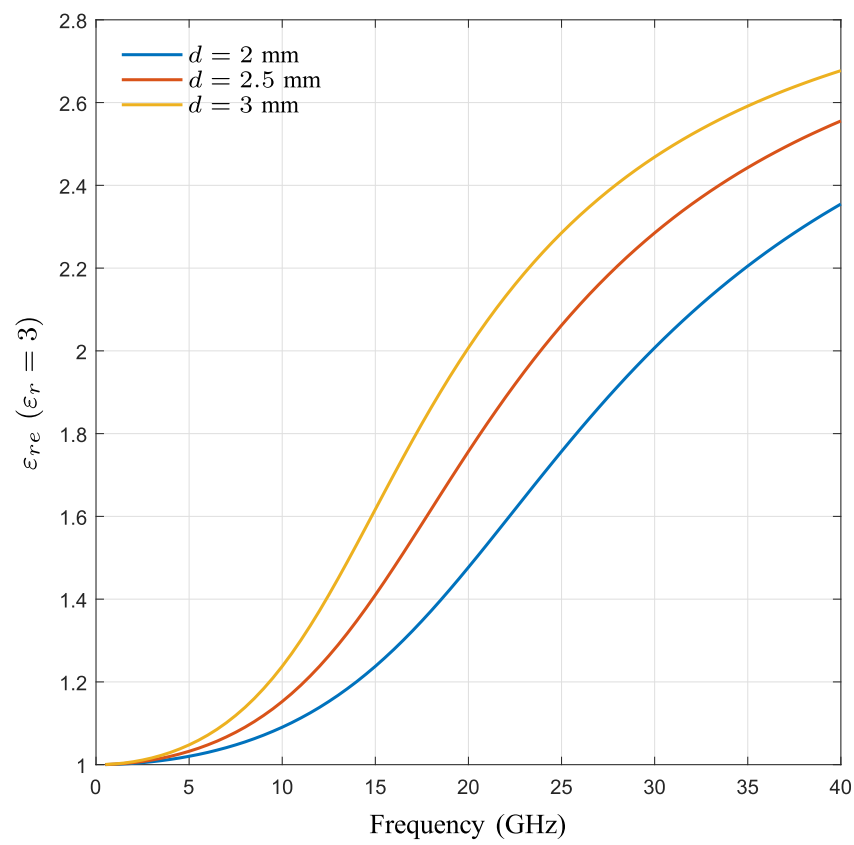

(b)

Fig. 3. Frequency-dependent effective permittivity. (a) Variation as a function of substrate permittivity, for a substrate thickness of $3 \mathrm{~mm}$. (b) Variation as a function of substrate thickness, for a substrate permittivity of 3 .

number; $k_{0}$ is the free-space wavenumber. The substrate thickness is $d$, its permittivity is $\varepsilon_{r}$, and the effective permittivity is $\varepsilon_{r e}$. The presence of $\tan (\cdot)$ makes this a transcendental equation that must be solved numerically; for this paper, a suitable routine was written in MATLAB. Fig. 3(a) and (b) shows how $\varepsilon_{r e}$ varies with frequency as a function of $\varepsilon_{r}$ (for a fixed $d$ ) and as a function of $d$ (for fixed $\varepsilon_{r}$ ), respectively. (We pre-empt Section III using values of $\varepsilon_{r}$ and $d$ relevant to the actual lens design in these figures. The value of $d$ used in Fig. 3(a) is the original design thickness, whereas those in Fig. 3(b) include the designed and fabricated thickness; see Section III-B. The value of substrate permittivity used in the example given in Fig. 3(b) has no significance other than being one of the design targets; equivalent plots can be generated for any other substrate permittivity.) It is important to note that the electric field is exponentially attenuated in region 2, with the attenuation determined by the frequency and the substrate permittivity and thickness. This surface wave confinement has implications for the design, in order to be able to measure and verify the fabricated lens performance.

Having computed the design curves, the effective permittivity curve can be extracted. To scale the GMFE curve appropriately requires the "background" substrate permittivity to be known; in this case, we took the permittivity of pure epoxy as 2.8 (giving $\varepsilon_{r e}=1.5263$ ), while $m=0.88$ (chosen to match the material and fabrication capabilities). For the third step, the design thickness was determined after consideration of the manufacturing tolerances in the selected casting process, together with the desired operating band and the degree of surface wave confinement (which has implications for later measurements). Having then computed the substrate curve, it was discretized following considerations of the degree of control over the permittivity of the composites and the tolerances in the casting process (due to the implications on the dimensions of the resultant rings). These factors are discussed further in the next section, with the final target permittivities given in Section III-B. We note that minimization of the resulting reflections between rings is also a factor in the discretization of the permittivity curve; the emphasis in this paper was on the realization of the device, rather than its optimization, so ensuring a final practical design that could be manufactured successfully within the achievable range of permittivities and their control was of primary concern in this respect.

\section{Composite Dielectrics AND DeVice FABRicAtion}

\section{A. Fabrication and Characterization of Composite Dielectrics}

Polymer-ferroelectric composites have been widely used for their electric properties in high frequency applications. Combining the advantages of the easy processability and high break down strength of polymers with the high dielectric constant of ferroelectric materials, polymer-ferroelectric composites offer a simple way to manufacture high permittivity materials with reproducible properties. Barium titanate $\left(\mathrm{BaTiO}_{3}\right)$ powder is the best studied ferroelectric that possesses a characteristic high dielectric constant. An epoxy matrix was chosen because of the relatively high dielectric constant of 2.8, as well as the ease of processability. By mixing $\mathrm{BaTiO}_{3}$ (BT) powders into epoxy matrix in specific ratios, the dielectric properties of the composites can be customized.

Micrometer-sized BT particles (smaller than $3 \mu \mathrm{m}$ ) supplied by Sigma Aldrich were chosen as the ceramic fillers, and a four-component Agar 100 Resin kit as the epoxy supply. The epoxy matrix material was prepared by mixing four prewarmed (at $60^{\circ} \mathrm{C}$ ) components (resin, hardeners, and accelerator) in ratios as stated in the manufacturer instructions. Pipettes were used for accurate measuring. Ceramic powders were then weighed and added into the epoxy matrix by volume fraction. 


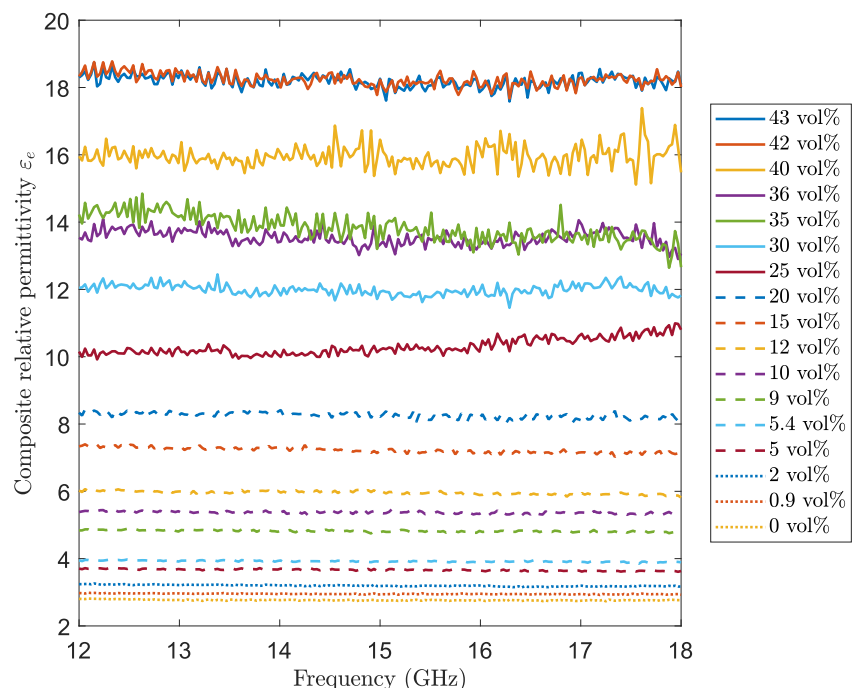

Fig. 4. Measured permittivity of epoxy/barium titanate composites with respect to frequency, for different volume-percentages of barium titanate (measured using a waveguide test fixture and the Nicolson-Ross-Weir extraction method).

The epoxy composite pastes were hand-stirred vigorously for approximately $30 \mathrm{~min}$ until well-dispersed mixtures were achieved, after which an ice-water bath sonication was used for about one h, to facilitate better dispersion. Composites with ceramic loadings of less than 30 vol\% were put into a vacuum oven under $0.1 \mathrm{MPa}$ at room temperature, to remove any trapped air. Composites with higher loadings exhibited too high a viscosity to be vacuum degassed. The degassed composites were poured into PTFE molds and put into a furnace to cure at $60{ }^{\circ} \mathrm{C}$ for at least $24 \mathrm{~h}$. Epoxy/BT composites with BT loadings from 0 to $43 \mathrm{vol} \%$ were prepared to explore the capability of producing composites with controllable dielectric properties.

The dielectric properties of the epoxy/BT composites were determined using samples measuring $15.8 \mathrm{~mm} \times$ $7.9 \mathrm{~mm} \times 2 \mathrm{~mm}$, for waveguide measurements at $\mathrm{Ku}$-band (12-18 GHz). An Agilent vector network analyzer (VNA) was used to measure the S-paramenters of a waveguide filled with the sample. The complex permittivity was determined from the (de-embedded) S-parameters using MATLAB, based on the Nicolson-Ross-Weir technique [55], [56] and assuming unity permeability. Up to five samples for each composition with BT loadings in the range $0-43$ vol\% were prepared and measured.

Fig. 4 shows the measured permittivity for samples of epoxy/BT composites with varying amounts of BT, over the frequency band of interest. It is clear that a nondispersive, stable dielectric constant was achieved for all epoxy/BT composites in the tested frequency range. The permittivity of the epoxy/BT composites steadily increased from around 3 to over 18 as more BT was added, as expected. Loss tangent values from around 0.03 to 0.12 were obtained in the waveguide measurements for these epoxy/BT composites. It is observed that the air bubbles contained in nondegassed high-loading composites show greater scattering, whereas good data consistency was obtained for measurements from different samples

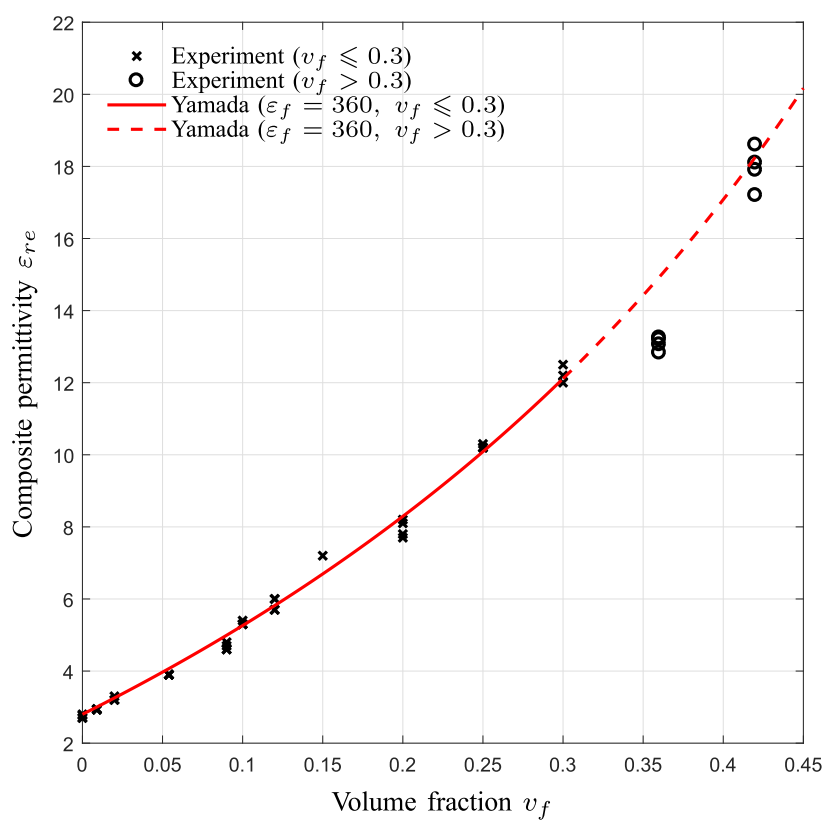

Fig. 5. Experimentally determined composite permittivities as a function of volume fraction at $15 \mathrm{GHz}$, with the Yamada curve (for $N=8.5, \varepsilon_{m}=2.8$, and $\left.\varepsilon_{f}=360\right)$.

with the same composition for degassed composites, which is suggestive of a more homogeneous and less dispersive distribution of the BT particles inside the epoxy resin.

The results at $15 \mathrm{GHz}$ were used to derive various relationships between volume fraction and composite permittivity. A number of models exist for this purpose (see [57], [58]); in this case, it was found [59] that the Yamada equation [60] (an alternative form of the Maxwell-Garnett equation derived for ellipsoidal particles) was a good fit to the measured composite permittivity, as shown in Fig. 5. The Yamada equation is given by [60], [61]

$$
\varepsilon_{c}=\varepsilon_{m}\left[1+\frac{v_{f} N\left(\varepsilon_{f}-\varepsilon_{m}\right)}{N \varepsilon_{m}+\left(1-v_{f}\right)\left(\varepsilon_{f}-\varepsilon_{m}\right)}\right]
$$

where $v_{f}$ is the volume fraction of the filler, $\varepsilon_{c}$ is the composite permittivity, $\varepsilon_{m}$ is the matrix (background material) permittivity, and $\varepsilon_{f}$ is the filler (particle) permittivity. $N$ is the inverse depolarization factor for the particles, which depends on the geometry of the ellipsoidal particles [57]. In practice, although the developed theory [57], [60] assumes $N$ is related only to the extent of deviation from sphericity of the minority inclusion (BT in this case), it is more realistic to treat it as a fitting parameter that also accounts for the likely inhomogeneities in the microstructure, such as limited agglomeration, clustering, alignment, and so on.

In this paper, two parameters were fixed: $\varepsilon_{m}=2.8$ (the relative dielectric permittivity of pure epoxy) and $N=8.5$ (an empirically determined value, consistent with previous reports of other similar-sized BT-containing polymeric composites [62] and other mixtures [60], [63]-[66]). After fitting, the BT relative dielectric permittivity was estimated as $\varepsilon_{f}=360$, with a $\chi^{2}$ tolerance of $1 \times 10^{-9}$. These values lie within those typically reported for the same materials and provided an acceptable fit to the data that allowed composites 


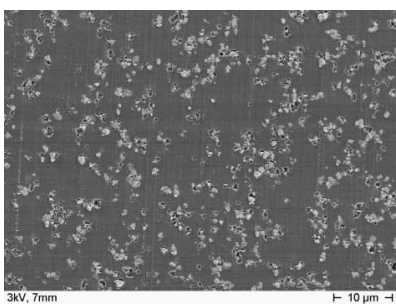

(a)

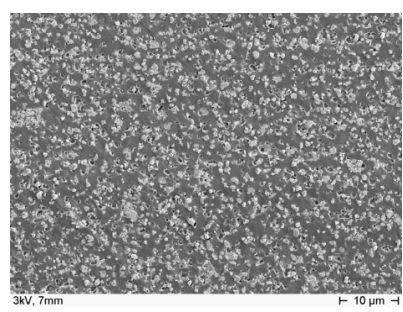

(c)

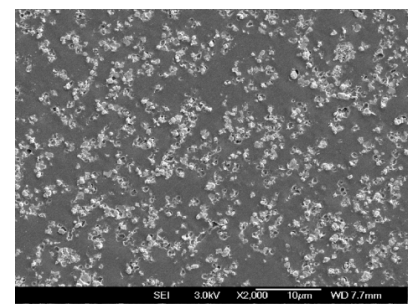

(b)

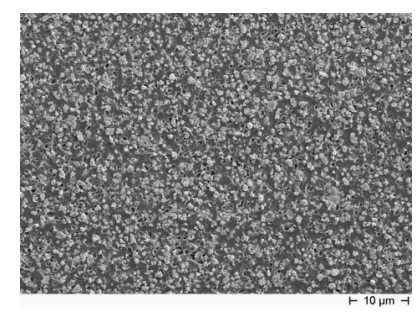

(d)
Fig. 6. SEM photographs of epoxy/BT composites for various volume fractions of barium titanate. (a) $5 \%$, (b) $15 \%$, (c) $25 \%$, and (d) $35.5 \%$.

of desired permittivity to be obtained simply by varying the BT volume fraction (see [64], [67], [68]). As only composites with BT volume ratios less than $30 \%$ were degassed (hence, there were strictly two component systems without air), only they were adopted for data fitting purposes. It is clear from Fig. 5, however, that the permittivity of nondegassed composites was broadly in line with the predicted values using the equation and values above.

To examine the microstructure of the epoxy/BT composites, scanning electron microscopy (SEM) images of the cross sections of epoxy/BT composites with a range of compositions were taken (with examples shown in Fig. 6), all of which revealed a homogeneous microstructure in the epoxy/BT composites.

\section{B. Fabrication of the Power-Dividing Lens}

Having determined the range of permittivities available and assessed the tolerances in the various stages of material and device fabrication, the GMFE lens profile was generated by setting $m=0.88$, with $d=3 \mathrm{~mm}$ and $f=15 \mathrm{GHz}$. This was then discretized into eleven concentric rings with relative permittivity values in ranging between 2.8 and 17 . More rings would improve the lens performance but increase the difficulty in implementation. As a proof-of-concept device, 11 rings were considered sufficient to demonstrate the functioning design while exploring the challenges around material development and fabrication.

The dielectric lens was manufactured by in situ casting using sets of concentric PTFE molds (see Fig. 7). The set of molds consisted of a 20-mm thick PTFE base with a 2-mm radius hole drilled in the middle, eleven 3-mm-thick PTFE layers with through holes in the middle, with radii from 2 to $80 \mathrm{~mm}$ in accordance with the design, and a 5-mm-thick aluminum plate on top that can be bolted to the PTFE base to clamp the PTFE layer in position during casting.

The lens was comprised of two parts: the central region contained the functional part, with the mapped graded permittivity; surrounding this was a dielectric background

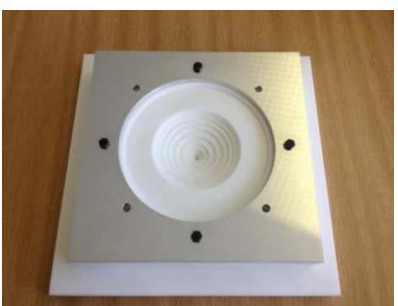

(a)

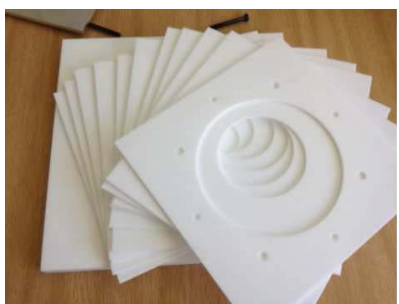

(b)
Fig. 7. Set of PTFE molds used to cast the device. (a) Assembled. (b) Individual.

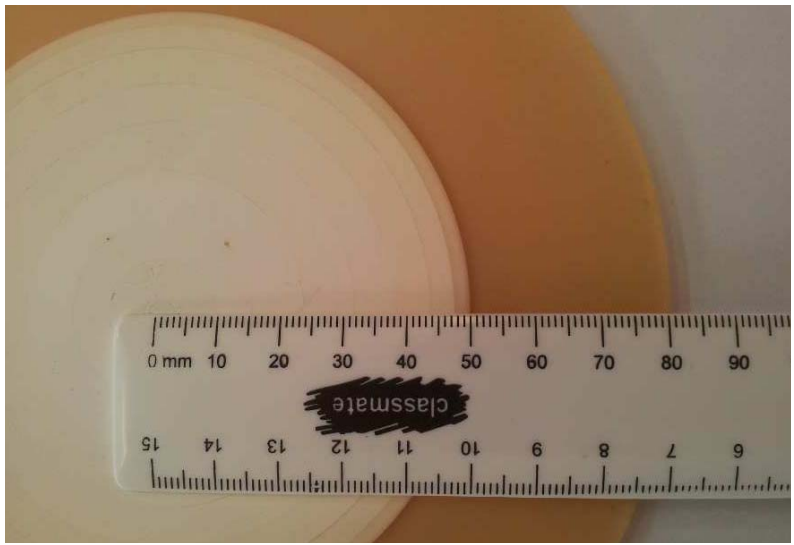

Fig. 8. Photograph of the lens showing visible interfaces between rings; the graded white region is the functional part of the lens, whereas the outer yellow region is the dielectric background.

made of pure epoxy resin, in which the propagation of the sub-beams could be observed after being split (see Fig. 8).

Well-mixed epoxy composites with volume fractions of BT powders between $0 \mathrm{vol} \%$ and $42 \mathrm{vol} \%$ were produced to give controllable permittivity from 2.8 to about 18 (as determined from Fig. 5). The casting process started from the innermost small section with the highest target permittivity of 17. This ring was cured in an oven at $60{ }^{\circ} \mathrm{C}$ for $24 \mathrm{~h}$ without degassing, due to its high BT volume fraction. Once it was fully cured, the PTFE layer was taken away, with the epoxy/BT composite piece anchored in the PTFE base for positioning purposes. Once the first ring was settled in place, the second ring composite material was poured into the second layer ring gap and gripped tightly onto the previous ring while curing. The rest of the lens was fabricated in the same fashion. Finally, the lens surfaces were machined in a lathe to be flat and parallel, with a final thickness of $2.5 \mathrm{~mm}$. This was thinner than the design; in consequence, the operating frequency shifted to $18 \mathrm{GHz}$, although the broadband nature of the device makes this of relatively low significance. This does show, however, that the design is robust to this type of fabrication error.

The final lens is shown in Fig. 8. The detailed dielectric profile for the individual rings of the lens is listed in Table I, where $r$ represents the outer radius of each ring, $\varepsilon_{r, t}$ is the target permittivity and $\varepsilon_{r, a}$ is the achieved permittivity.

In general, there was very good interfacial cohesion between the rings and, although the boundaries between 
TABLE I

Target and Mean Achieved Permittivity Profile of the LENS, With VOLUME Fractions AND ACHIEVEd Loss TANGEnTS. Note: THE MEASUREMEnT ERRor $\left(\Delta \varepsilon_{r, a}\right)$ WAS $+1 \%-0.2 \%$ FOR ALL SAMPLES

\begin{tabular}{rrrrrr}
\hline Ring & $r(\mathrm{~mm})$ & Volume \% BT & $\varepsilon_{r, t}$ & $\bar{\varepsilon}_{r, a}$ & $\tan (\delta)$ \\
\hline \hline 1 & 2.0 & 42.0 & 17.00 & 17.95 & 0.10 \\
2 & 4.6 & 36.0 & 14.00 & 13.09 & 0.09 \\
3 & 8.6 & 30.0 & 12.00 & 12.23 & 0.07 \\
4 & 14.7 & 25.0 & 10.00 & 10.23 & 0.09 \\
5 & 22.9 & 20.0 & 8.00 & 7.95 & 0.06 \\
6 & 30.9 & 12.0 & 6.00 & 5.85 & 0.08 \\
7 & 37.2 & 9.0 & 5.00 & 4.68 & 0.05 \\
8 & 43.0 & 5.4 & 4.00 & 3.90 & 0.05 \\
9 & 46.8 & 2.0 & 3.40 & 3.23 & 0.04 \\
10 & 49.2 & 0.9 & 3.00 & 2.94 & 0.03 \\
11 & 80.0 & 0.0 & 2.77 & 2.73 & 0.03 \\
\hline
\end{tabular}

individual rings were clearly visible, there were no air gaps or cracks (Fig. 8). During the casting process, the same materials used to manufacture the rings of the lens were also used to make several $15.8 \mathrm{~mm} \times 7.9 \mathrm{~mm} \times 2 \mathrm{~mm}$ block samples for waveguide measurements at $\mathrm{Ku}$-band (12-18 GHz). As seen in Table I $\left(\varepsilon_{r, a}\right)$, satisfactory control was achieved over the dielectric constant of epoxy composite materials in meeting the target values. The loss tangent for these epoxy/BT composites was between 0.03 and 0.12 , as seen in Section III-A.

\section{Measurements And Discussion}

\section{A. Initial Measurement Campaign}

The lens was tested in a near-field imaging system at the University of Exeter, consisting of a two-port VNA, monopole transmitter and receiver, a metal base on which the lens was sitting (ideally in perfect contact), and some absorbing materials surrounding the lens (see Fig. 9). The monopole receiver was placed immediately above the interface between the lens and the dielectric background at $z=3 \mathrm{~mm}$, which was thus $0.5 \mathrm{~mm}$ away from the lens surface. The scan was performed over an area of $190 \mathrm{~mm} \times 190 \mathrm{~mm}$ at a step size of $1 \mathrm{~mm}$, between $15 \mathrm{GHz}$ and $20 \mathrm{GHz}$. Unfortunately, some warping of the lens had occurred between fabrication and testing. The warping arises because each of the concentric rings has a slightly different coefficient of expansion to its neighbor because of the varying BT fraction. During the thermal cycling as each layer is cast and cured, differential thermal expansion/contraction evolves small residual strains in the rings, making some distortion difficult to suppress. Optimizing these thermal cycles and/or the addition of a final extended annealing treatment and slow cooling would likely reduce these strains. Further, the strains are relatively easily manifest as distortions in this relatively thin, large area device, and would be less noticeable in thicker variants, or in 3-D analogs. Here, the lens was compressed for around $24 \mathrm{~h}$ before measurements were made, but an air gap beneath the lens could not be removed completely. Measurements proceeded,

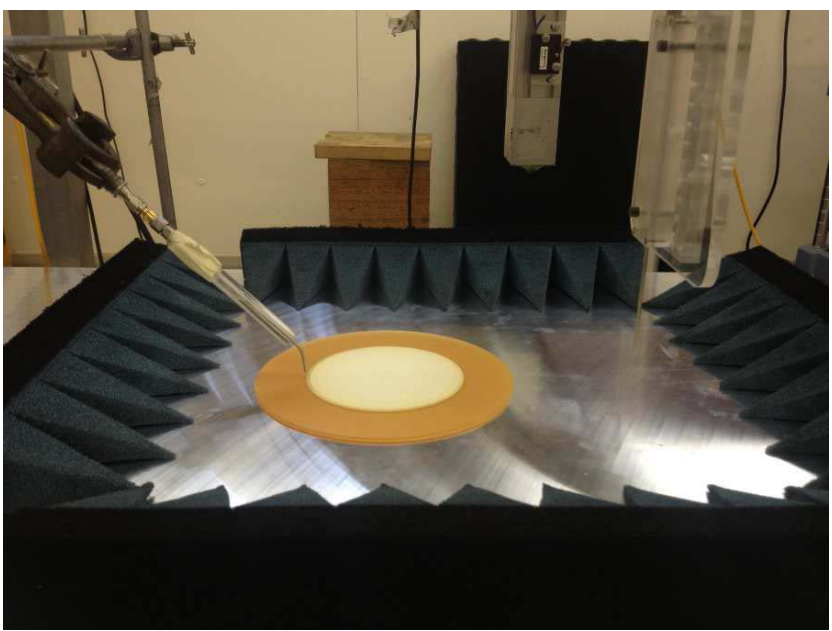

Fig. 9. Photograph of the lens testing environment at the University of Exeter.

both because of the time and effort involved in fabrication, and to see the robustness of the lens functionality to the air gap.

The measured electric field $\left(E_{z}\right)$ amplitude and instantaneous $E_{z}$ at $18 \mathrm{GHz}$ are plotted in Fig. 10. The spherical wave from the monopole transmitter was first converted into local plane wave in the first half of the lens before the wavefront was curved again toward the second half. Beam splitting, with beams emanating from similar positions as in the simulation, is detected in the dielectric background, as well as in air. However, the feature of the initiation of the two split beams in the second half of the lens is not as obvious in the measurements as in the simulations of Fig. 2(b). There are two possibilities:

1) the power division (dual focal points) occurs as expected, but at a low magnitude that makes it difficult to see against other factors (e.g., background noise, reflections);

2) the lens is not functioning as predicted by the design, presumably due to the air gap.

To confirm that the fabricated lens should have functioned correctly (had warping not occurred), a 3-D model simulating the discrete lens performance was constructed in COMSOL Multiphysics using the RF module, including the discrete lens and a model of the coaxial monopole source.

The lens sat on the $x-y$ plane with its bottom center located at the origin. A perfect electric conductor was chosen for geometrical boundary at $z=0 \mathrm{~mm}$, representing the metallic base in the actual test. A coaxial cable, with an inner conductor radius of $0.5 \mathrm{~mm}$ and outer conductor radius of $2.5 \mathrm{~mm}$, was constructed as the monopole transmitter, by exposing the inner conductor. The exposed end of the monopole was placed $0.5 \mathrm{~mm}$ above the lens (i.e., $z=3 \mathrm{~mm}$ ), right above the interface between the 10th and 11th rings of the lens (i.e., between the dielectric background and the functional lens). A hemispherical perfectly matched layer (PML) domain, with an outer radius of $165 \mathrm{~mm}$, was employed, situating outside the air domain to absorb incident radiation without producing reflections. A free tetrahedral mesh was applied 


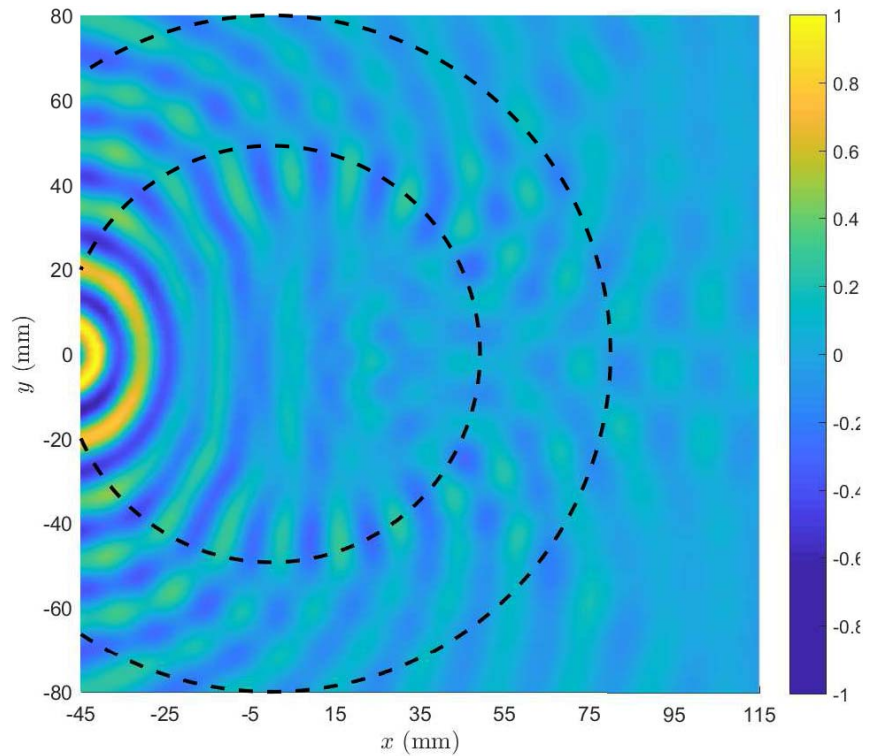

(a)

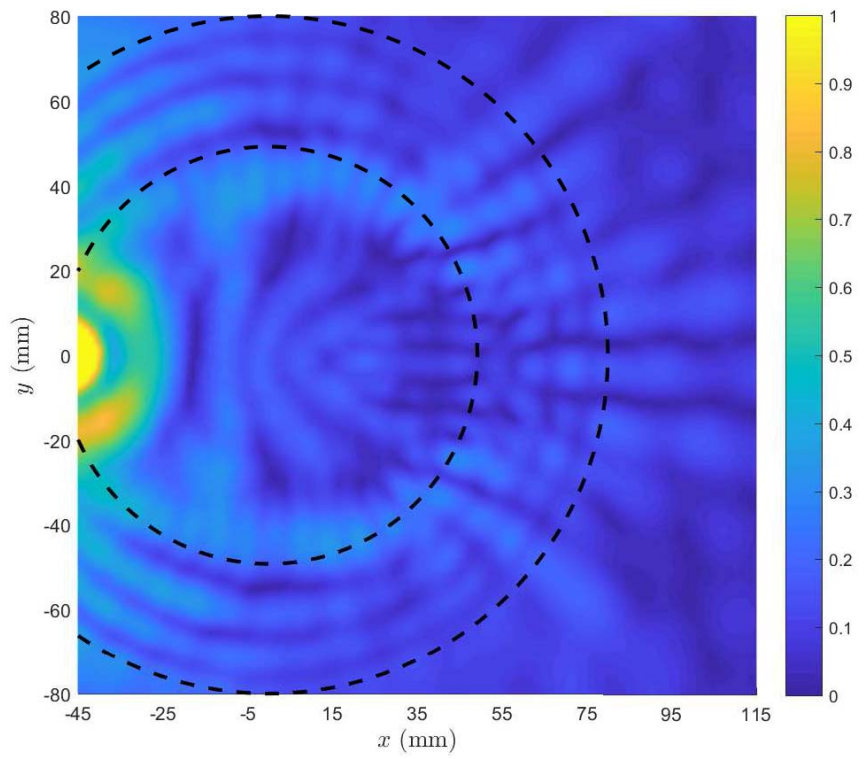

(b)

Fig. 10. Normalized measured $z$ component of the electric field $\left(E_{z}\right)$ at $18 \mathrm{GHz}$. (a) Instantaneous $E_{z}$. (b) $\left|E_{z}\right|$. The two dashed overlays mark the outer extent of the lens and the dielectric background.

to all domains (with the exception of the PML), with the maximum element size calculated as one fifth of the local wavelength. Measured dielectric properties, including the loss tangents of all dielectric rings of the lens, were used to specify the materials properties in these simulations to replicate the real lens testing environment as closely as possible.

The simulated electric field $\left(E_{z}\right)$ distributions at the cut plane of $z=3 \mathrm{~mm}$ and at $18 \mathrm{GHz}$ are plotted in Fig. 11 . Both the $E_{z}$ amplitude and instantaneous $E_{z}$ plots show how the spherical wave was first converted into a local plane wave at the beginning of its propagation and then bent into two split beams (instead of one spherical wave, as in a standard MFE lens) at the lens boundary on the opposite side. (We note that the measurement difficulties preclude experimental demonstra-

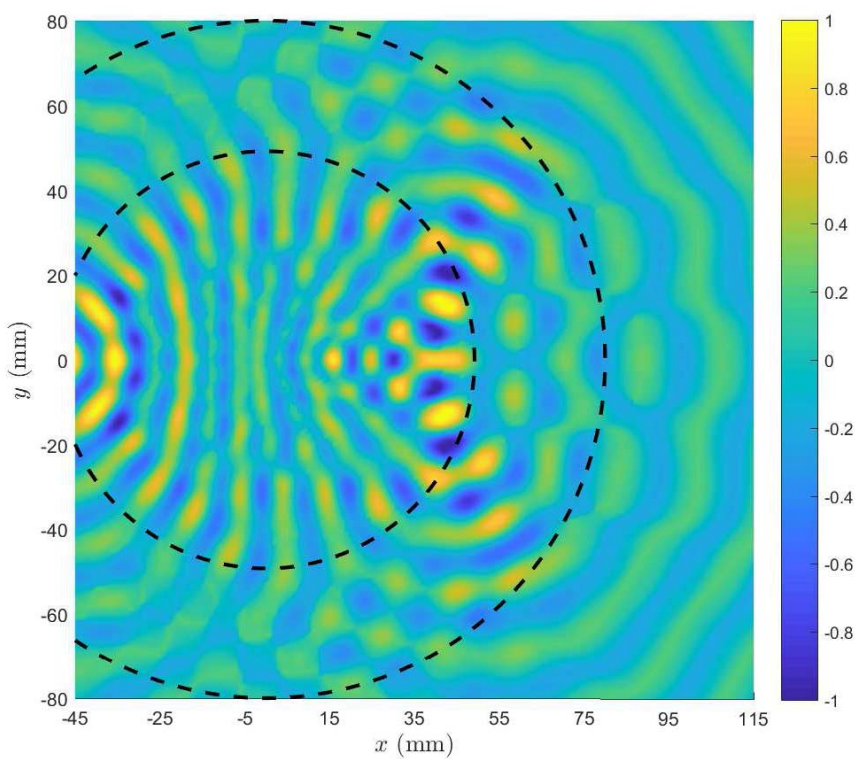

(a)

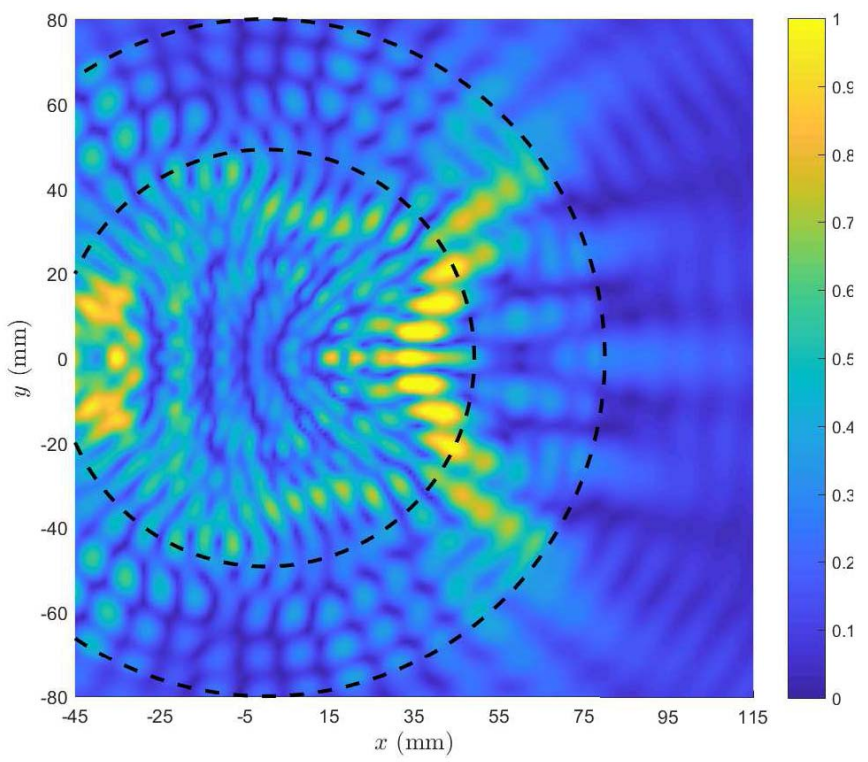

(b)

Fig. 11. Normalized electric field $z$ component $\left(E_{z}\right)$ at $18 \mathrm{GHz}$, simulated with COMSOL. (a) Instantaneous $E_{z}$. (b) $\left|E_{z}\right|$. The two dashed overlays mark the outer extent of the lens and the dielectric background.

tion of broadband operation at this time; we refer the reader to Appendix A, where further results from the full-wave simulations of the discrete lens are shown for other frequencies, demonstrating the expected broadband beam-splitting behavior. We emphasize that the measurements showed broadband behavior, in that similar deviations in focusing from the design were observed across the band.) As the COMSOL simulation included the loss without affecting the ability of the lens to produce two beams, this possible explanation was ruled out as a factor.

\section{B. Effect of the Air Gap}

As stated above, a small nonuniform gap thus existed below the lens, being approximately $1.0 \mathrm{~mm}$ in the center and 
$0 \mathrm{~mm}$ at the outer edges. This gap would reduce the effective permittivity, affecting the ability of the lens to focus the input. The larger gap occurred in the portion of the GMFE lens where it would have most effect (Fig. 1). As it was not possible to get an accurate measurement of the gap profile, an alternative means of investigation was sought.

First, simulations were performed using 2-D models (for speed) in COMSOL. Due to the difficulty of modeling the induced curvature of the lens, this was achieved via changes to the permittivity and loss tangent of each ring. This was done on a qualitative basis, in order to see if results resembling the experimental data could be produced. The changes to the permittivity profile were generated using a simple model, with the rotationally symmetric air gap $h$ given by

$$
h(r)=h_{0}\left[1-\left(\frac{r}{r_{g}}\right)^{2}\right]
$$

where $h_{0}=(1 \mathrm{~mm})$ is the maximum air gap and $r_{g}(=50 \mathrm{~mm})$ is the radial position where the gap ceases. This inverted quadratic curve was selected as it is the simplest nonlinear model with the largest air-gap in the center of the lens.

The new permittivity $\varepsilon_{g}$ was then generated from the old permittivity $\varepsilon_{r}$ using a simple proportional basis

$$
\begin{aligned}
A & =\frac{d}{d+h} \\
B & =1-A=\frac{h}{d+h} \\
\varepsilon_{g} & =A \varepsilon_{r}+B
\end{aligned}
$$

where $d(=2.5 \mathrm{~mm})$ is the substrate thickness of Section II. These equations were applied to the discrete design using $r_{i}$ and $\varepsilon_{r, a}$ from Table I, for $i \in[1,10] \cap \mathbb{N}$. While this approach is undoubtedly simplified (the assumption of a rotationally symmetric quadratic for the gap profile, the choice of $r_{g}$, the proportionality method, plus the 2-D simulation method), it provided a fast and easy means of generating and simulating a structure to see how the beam formation was affected.

As seen in the example plot given in Fig. 12, the two strong beams from the original distribution have been replaced with multiple beams, although the behavior in the central region still differs to that observed in the experimental data. As the simulation was a 2-D model of bulk dielectrics (not surface waves) and did not include loss, the strength of the beams is unsurprisingly still relatively large. The agreement is also imperfect in terms of the beam positions and other details (unsurprising, since the permittivity changes may not fully represent the effect of the gap, in terms of both their magnitudes and also how the wave propagates in a 3-D scenario), yet it is suggestive that the air gap caused by the warping could account for the observed differences in beam generation between prediction and experiment.

It is worth noting that the ability to provide more than two-way power division is of considerable interest; this demonstration is therefore serendipitous in offering a route toward modifying GMFE lenses to achieve such functions. This is discussed further in Appendix B.

Second, a newly available surface scanning technique was used to map the permittivity of the fabricated lens.

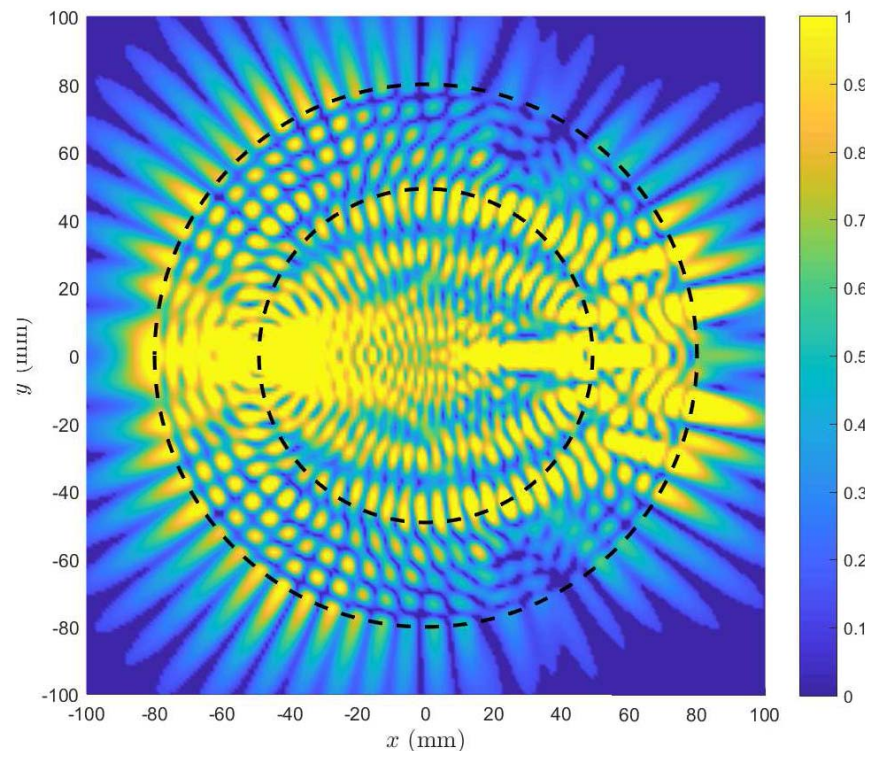

Fig. 12. Normalized absolute electric field $z$ component $\left(\left|E_{z}\right|\right)$ of the modified permittivity distribution at $18 \mathrm{GHz}$, simulated with COMSOL; the two dashed overlays mark the outer extent of the lens and the dielectric background (other rings not shown).

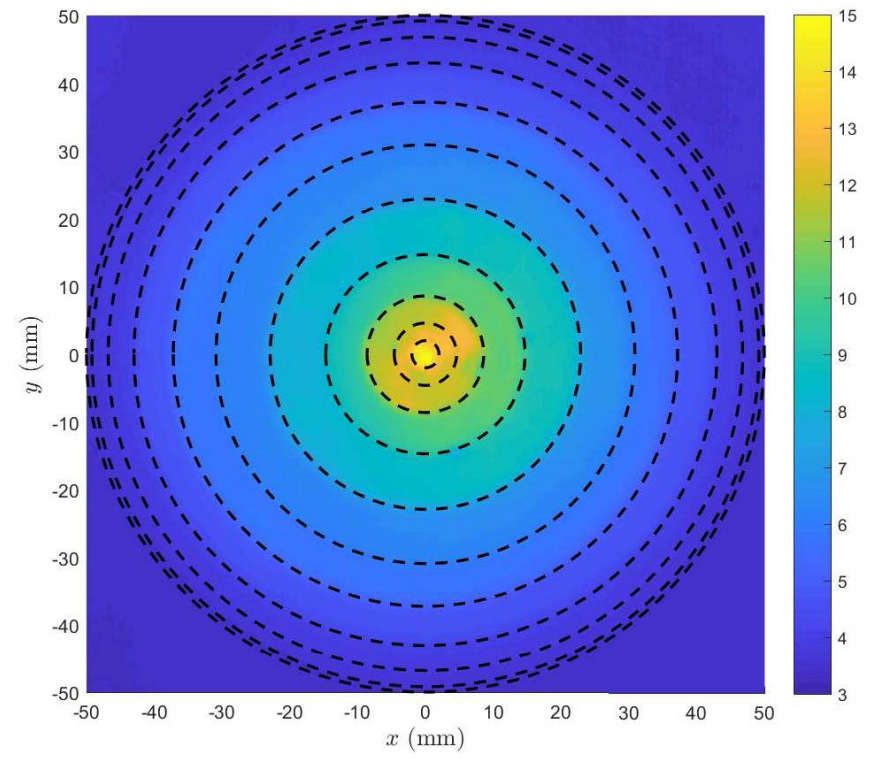

Fig. 13. Measured permittivity of the GMFE lens using an SRR probe and a surface scanning method. (The dashed overlays for the rings were not required to compute the permittivity but have been added to demonstrate the accuracy of the technique in detecting local variation in permittivity.)

This technique was developed relatively recently, and has been used to map the permittivity of both anisotropic layered composite structures [22] and a layered lens [69]. It will be fully described in a forthcoming publication [70]. Briefly, the spatial distribution of the dielectric permittivity was measured using a split ring resonator (SRR) probe in contact with the lens surface, moving over the surface of the lens in discrete steps. A circuit model was used to estimate the effect of the (local) permittivity on the resonant response of the SRR probe. A spatial resolution between 0.1 and $2 \mathrm{~mm}$ can be achieved, with measurement errors of up to $10 \%$ in permittivity. 
The spatial mapping of the permittivity of the warped lens is shown in Fig. 13, with the rings overlain for clarity (these were not required to compute the permittivity but have been added to demonstrate the accuracy of the technique in detecting local variation in permittivity). It is evident that the (effective) permittivity values are different from the achieved values measured by the waveguide test fixture; this is attributed to the air-gap beneath the lens. The (effective) permittivity in the central region is not as low as suggested by the model in (8), which suggested a maximum of 12.6. This can be partly explained by the simplifications and assumptions of the model being (somewhat) inaccurate. Another potential factor could be imperfect contact of the SRR probe to the curved surface of the warped lens. Some slight inhomogeneity can be seen in some of the rings; this could reflect the presence of an asymmetric air-gap, or possibly even inhomogeneities in the dielectric rings. Comparing the scanned values with those in Table I, it is clear that the central rings have a lower (effective) permittivity than required, as expected from the air-gap.

\section{CONCLUSION}

In this paper, we have provided a case study of how an all-dielectric transformation electromagnetics device can be designed, realized, and experimentally validated. Specifically, an all-dielectric GMFE lens for surface waves was fabricated by a simple, low-cost, and geometrically flexible casting route using epoxy/barium titanate composites. The precision control over the permittivity of the epoxy composites is crucial in achieving the beam splitting function offered by the GMFE lens. For surface wave devices, additional fabrication challenges, such as differential thermal expansion/contraction in graded materials, emphasizes the need to apply careful process control if such devices are to be realized by industry. The measured electric field over the lens showed partial splitting of beams that emanate from the lens in broad qualitative agreement with simulation. While some differences were observed between the fabricated lens and the original design, further improvements in the fabrication process can be made to avoid warping, including optimization of the manufacturing thermal history, post manufacture annealing, and geometrical changes to increase stiffness and the resistance to distortion. It is noted that neither a bulk lens nor a thin parallel-plate waveguide lens would be as sensitive to the warping observed in the surface wave lens.

As a proof-of-concept device, the GMFE lens demonstrated a simple, cost-efficient, well-established, and geometrically flexible casting method to fabricate all-dielectric GRIN devices with good control of dielectric properties. While the GMFE lens discussed in this paper did not utilize TrE directly, transformations of the lens shape are readily accommodated, allowing (for example) flattened input and output surfaces (see [19]). The GMFE lens acts as a useful prototype for a broadband, isotropic beam splitter (Appendix A); when combined with (quasi-conformal) TrE and optimization, it is expected that more directly useful geometries can be achieved without imposing excessive material requirements. Furthermore, it is possible that unequal power division, or beam

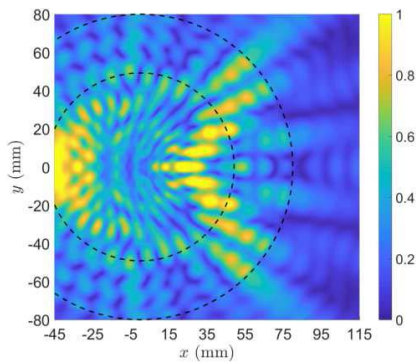

(a)

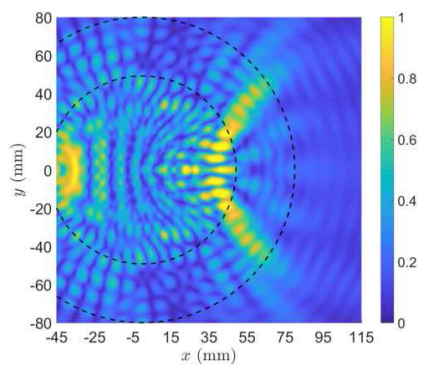

(c)

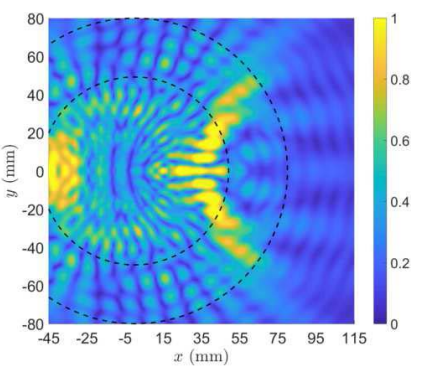

(b)

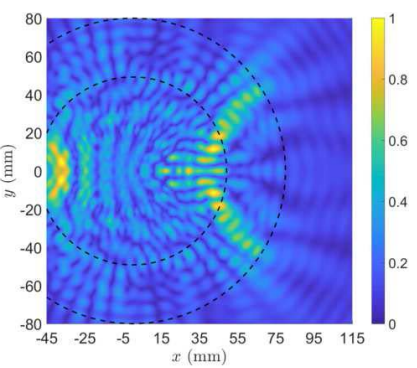

(d)
Fig. 14. COMSOL simulations showing broadband beam splitting for the discrete surface wave lens (scales as in Fig. 11). (a) 15, (b) 17, (c) 19, and (d) $20 \mathrm{GHz}$.

numbers greater than two, or even both of these, could be achieved by modifying the GMFE profile. (Although it was not possible to discuss this in any depth in this paper, some comments on this and other advanced issues are given in Appendix B.)

Finally, it has been suggested that the implementation of TrE designs using an all-dielectric approach is viable, with a range of controlled permittivities and fabrication methods now available. The improvements to realize practical devices of the power divider type, operating close to a design specification, are relatively straightforward and practical, and suggest that all-dielectric $\operatorname{TrE}$ devices could be achieved on a larger and industrially relevant scale relatively easily.

\section{APPENDIX A}

\section{Simulated BROADBAND OPERATION}

One of the motivations for using an all-dielectric approach is to increase the bandwidth of operation. Due to the issues discussed in Section IV, it was not possible to fully explore this behavior for the fabricated lens. Hence, we present in Fig. 14 a small selection of simulation results demonstrating beam splitting at frequencies away from the design frequency of $18 \mathrm{GHz}$, using the 3-D COMSOL model from Section IV.

\section{APPENDIX B \\ ADVANCED ISSUES}

It is desirable to provide arbitrary power division, both in terms of the number of output beams and in terms of the power ratios of the output beams. The ideal GMFE lens alone provides two output beams with equal power. In practice, a number of undesirable secondary beams can be observed in the simulated results of Section IV-A. These are a result of at least three factors: 


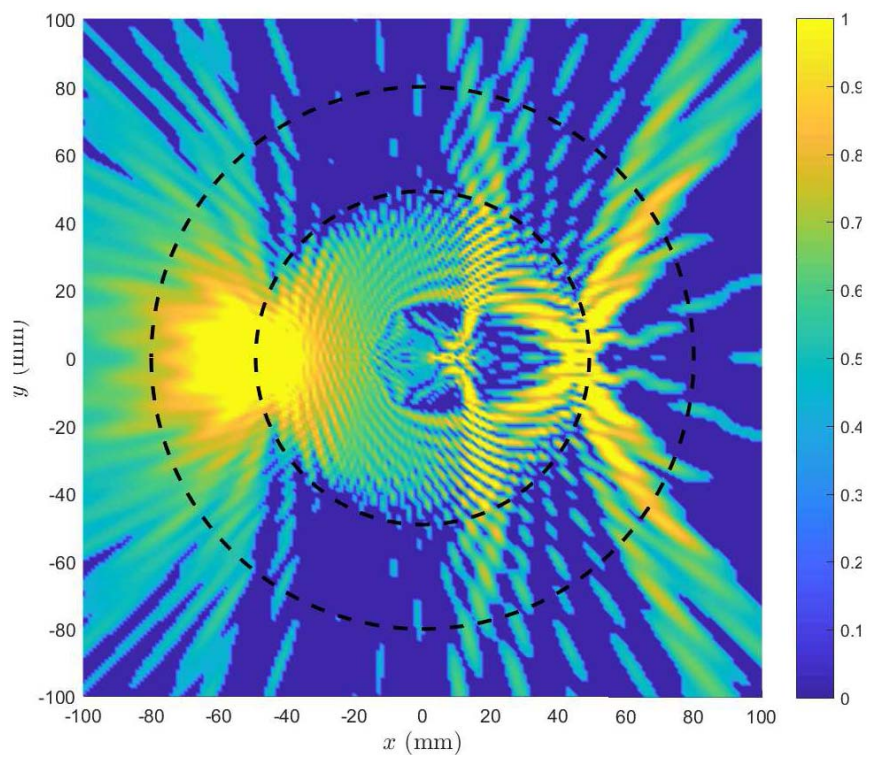

Fig. 15. Example of combining two GMFE lenses (black dashed circles) to increase the number of beams, simulated in COMSOL: normalized $\left|E_{z}\right|$ with low magnitude signals suppressed $\left(f=50 \mathrm{GHz}, m_{\text {outer }}=0.95\right.$, $m_{\text {inner }}=0.75, r_{\text {outer }}=50 \mathrm{~mm}, r_{\text {inner }}=15 \mathrm{~mm}$, and assuming a background of air with $\left.\varepsilon_{\text {outer }}\left(r \geqslant r_{\text {outer }}\right)=1\right)$.

1) the nondirective monopole source;

2) the relatively small electrical size of the device (six free-space wavelengths in diameter at $18 \mathrm{GHz}$ );

3) reflections from the layers in the discretized profile.

Improvements are expected with refinements in these areas, with some limitations (in particular, diffraction could result in power in the region between the beams [4]). It is possible, however, that these secondary beams could be the basis for a beam splitter with more than two beams, perhaps using optimization of the discretized lens.

Alternatively, the experimental results (Section IV-A) and investigation into the effects of an air-gap (Fig. 12) both show how variations in the permittivity profile from the GMFE profile can result in multiple beams. The simple quadratic model of the air gap suggests that various models for changing the permittivity could be explored with relative ease. The challenge here would be to have a means of specifying the number of beams and, ideally, the power division ratios between them. Controlling the position of the beams would also be desirable, although this could be achieved to some extent via additional use of TrE. It is noticeable that the beams of the modified GMFE lens (Fig. 12) are clustered on the opposite side of the lens to the feed.

One possible method to control the number (and position) of beams could be to combine two (or possibly more) GMFEbased lenses into one lens. As seen in Fig. 1, there is little difference between profiles of differing $m$ in the outer regions of the lens. By replacing the profile for the central region of one lens (say, with $m=0.95$ ) with the profile of a different lens (say, $m=0.75$ ), the portion of the wave passing through the central region is focused differently to those in the outer region. Careful control of the region size and values of $m$ would be necessary to realize a practical and efficient lens. As an illustration of this concept, we show in Fig. 15 the results from a 2-D study in COMSOL with four main beams (compare with the beam positions in Fig. 2 for these values of $m$ ). The permittivity outside the lens (outer dashed circle) is free space, for simplicity; the permittivities of the two lens regions were calculated using (1) without discretization or scaling, for these two values of $m$. A coaxial monopole was used as the source, as in the previous simulations. In order to show the behavior clearly, the frequency was increased to $50 \mathrm{GHz}$, effectively increasing the electrical size of the lens, and low magnitude signals were suppressed (a more directive source should improve the lens performance for lower frequencies). The power ratios of the beams were not controlled directly, though it is possible that optimization techniques could be used to achieve this with a combined GMFE lens as the initial design. Optimization can also be used with a discretized version of the combined GMFE lens directly. (We note again that TrE could be used to make the output beam surfaces flat, and even further separate the beams spatially if necessary; collimation of the diverging beams can also be included.)

An alternative method to control the power ratio is to utilize a spatial variation in loss (or gain, or both), via complex coordinate TrE. A wholly loss-based solution would be simpler to fabricate, but suffer from reduced efficiency.

It should also be noted that the GMFE lens does not combine two incident beams into one output beam. Rather, each input beam is divided (into two, for a standard GMFE lens) and a given split beam for one input can be combined with one split beam per other input, achieved by careful control of incident angles given a specific GMFE profile. Thus, the GMFE lens does not function efficiently as a power combiner without modification, due to the "wasted" power in the uncombined output beams. On the other hand, the GMFE lens can, in principle, independently divide multiple inputs simultaneously, as long as they are positioned such that the split outputs do not coincide. This could be on a grouped basis, such that the outputs from one input are adjacent, or interleaved, dependent on the angular separation(s) of the input beams for a given GMFE profile.

\section{ACKNOWLEDGMENT}

The authors would like to thank the assistance of Prof. C. Parini (QMUL), Prof. O. Quevedo-Teruel (formerly with QMUL; now with the KTH Royal Institute of Technology, Sweden), and Dr. L. Zhang (formerly with QMUL). The authors would like to thank the assistance of Dr. D. Isakov (University of Oxford) in measuring the surface permittivity of the lens. The authors would also like to thank the assistance of Dr. J. Dockrey (formerly with the University of Exeter) in initial measurements. Valuable conversations with Dr. R. Mitchell-Thomas (formerly with QMUL; now with University of Exeter) are also noted.

\section{REFERENCES}

[1] D. Schurig et al., "Metamaterial electromagnetic cloak at microwave frequencies," Science, vol. 314, no. 5801, pp. 977-980, Nov. 2006.

[2] U. Leonhardt, "Notes on conformal invisibility devices," New J. Phys., vol. 8 , no. 7 , p. 118, Jul. 2006.

[3] J. Li and J. B. Pendry, "Hiding under the carpet: A new strategy for cloaking," Phys. Rev. Lett, vol. 101, no. 20, Nov. 2008, Art. no. 203901. 
[4] M. Rahm, D. A. Roberts, J. B. Pendry, and D. R. Smith, "Transformation-optical design of adaptive beam bends and beam expanders," Opt. Exp., vol. 16, no. 15, pp. 11555-11567, Jul. 2008.

[5] W. X. Jiang, T. J. Cui, X. Y. Zhou, X. M. Yang, and Q. Cheng, "Arbitrary bending of electromagnetic waves using realizable inhomogeneous and anisotropic materials," Phys. Rev. E, Stat. Phys. Plasmas Fluids Relat. Interdiscip. Top., vol. 78, no. 6, Dec. 2008, Art. no. 066607.

[6] Z. L. Mei and T. J. Cui, "Experimental realization of a broadband bend structure using gradient index metamaterials," Opt. Exp., vol. 17, no. 20, pp. 18354-18363, Sep. 2009.

[7] D.-H. Kwon and D. H. Werner, "Polarization splitter and polarization rotator designs based on transformation optics," Opt. Exp., vol. 16, no. 23 , pp. 18731-18738, Nov. 2008

[8] M. I. Stockman, "Criterion for negative refraction with low optical losses from a fundamental principle of causality," Phys. Rev. Lett, vol. 98 , no. 17, Apr. 2007, Art. no. 177404.

[9] T. Tyc, L. Herzánová, M. Šarbort, and K. Bering, "Absolute instruments and perfect imaging in geometrical optics," New J. Phys., vol. 13, no. 11, Nov. 2011, Art. no. 115004.

[10] H. Chen, C. T. Chan, and P. Sheng, "Transformation optics and metamaterials," Nature Mater, vol. 9, no. 5, pp. 387-396, Apr. 2010.

[11] U. Leonhardt, "Optical conformal mapping," Science, vol. 312, no. 5781, pp. 1777-1780, Jun. 2006.

[12] J. B. Pendry, D. Schurig, and D. R. Smith, "Controlling electromagnetic fields," Science, vol. 312, pp. 1780-1782, Jun. 2006.

[13] D. E. Brocker, J. P. Turpin, P. L. Werner, and D. H. Werner, "Optimization of gradient index lenses using quasi-conformal contour transformations," IEEE Antennas Wireless Propag. Lett., vol. 13, pp. 1787-1791, 2014.

[14] H. F. Ma and T. J. Cui, "Three-dimensional broadband ground-plane cloak made of metamaterials," Nature Commun., vol. 1, p. 21, Jun. 2010.

[15] D. Bao et al., "All-dielectric invisibility cloaks made of $\mathrm{BaTiO}_{3}$ loaded polyurethane foam," New J. Phys., vol. 13, no. 10, Oct. 2011, Art. no. 103023

[16] M. Yin, X. Y. Tian, L. L. Wu, and D. C. Li, "All-dielectric threedimensional broadband Eaton lens with large refractive index range," Appl. Phys. Lett., vol. 104, no. 9, Mar. 2014, Art. no. 094101.

[17] M. Rahm, S. A. Cummer, D. Schurig, J. B. Pendry, and D. R. Smith, "Optical design of reflectionless complex media by finite embedded coordinate transformations," Phys. Rev. Lett, vol. 100, no. 6, Feb. 2008, Art. no. 063903. [Online]. Available: http://link.aps.org/doi/10.1103/ PhysRevLett.100.063903

[18] Y.-L. Wu, Z. Zhuang, L. Deng, and Y.-A. Liu, "Three-dimensional multiway power dividers based on transformation optics," Sci. Rep., vol. 6, Apr. 2016, Art. no. 24495. [Online]. Available: http://www.nature.com/ articles/srep24495

[19] D. R. Prado, A. V. Osipov, and O. Quevedo-Teruel, "Implementation of transformed lenses in bed of nails reducing refractive index maximum value and sub-unity regions," Opt. Lett., vol. 40, no. 6, pp. 926-929, Mar. 2015.

[20] Q. Lei, C. E. J. Dancer, C. R. M. Grovenor, and P. S. Grant, "Preparation, microstructure and microwave dielectric properties of sprayed PFA/barium titanate composite films," Compos. Sci. Technol., vol. 129, pp. 198-204, Jun. 2016. [Online]. Available: http://www.sciencedirect. com/science/article/pii/S0266353816301580

[21] F. Castles et al., "Microwave dielectric characterisation of 3D-printed $\mathrm{BaTiO}_{3} / \mathrm{ABS}$ polymer composites," Sci. Rep., vol. 6, Jan. 2016, Art. no. 22714. [Online]. Available: http://www.nature.com/srep/2016/ 160304/srep22714/full/srep22714.html

[22] D. V. Isakov, Q. Lei, F. Castles, C. J. Stevens, C. R. M. Grovenor, and P. S. Grant, "3D printed anisotropic dielectric composite with meta-material features," Mater. Des., vol. 93, pp. 423-430, Mar. 2016. [Online]. Available: http://www.sciencedirect.com/science/article/pii/ S0264127515310340

[23] L. Parke et al., "Heavily loaded ferrite-polymer composites to produce high refractive index materials at centimetre wavelengths," APL Mater. vol. 1, no. 4, p. 042108, Oct. 2013.

[24] Y. Wang, E. Edwards, I. Hooper, N. Clow, and P. S. Grant, "Scalable polymer-based ferrite composites with matching permeability and permittivity for high-frequency applications," Appl. Phys. A, Solids Surf., vol. 120, no. 2, pp. 609-614, Aug. 2015. [Online]. Available: http://link.springer.com/10.1007/s00339-015-9223-z

[25] Y. Wang, F. Castles, and P. S. Grant, "3D printing of NiZn ferrite/ABS magnetic composites for electromagnetic devices," in Proc. MRS , vol. 1788. Jul. 2015, pp. 29-35. [Online]. Available: http://journals.cambridge.org/abstract_S1946427415006612
[26] P. S. Grant et al., "Manufacture of electrical and magnetic graded and anisotropic materials for novel manipulations of microwaves," PhilPhilos. Mag. A, Phys. Condens. Matter Defects Mech. Prop., vol. 373, no. 2049, Aug. 2015, Art. no. 20140353. [Online]. Available: http://rsta. royalsocietypublishing.org/lookup/doi/10.1098/rsta.2014.0353

[27] J. A. Dockrey, M. J. Lockyear, S. J. Berry, S. A. R. Horsley, J. R. Sambles, and A. P. Hibbins, "Thin metamaterial Luneburg lens for surface waves," Phys. Rev. B, Condens. Matter, vol. 87, no. 12, Mar. 2013, Art. no. 125137. [Online]. Available: http://journals.aps.org/ prb/abstract/10.1103/PhysRevB.87.125137

[28] A. P. Hibbins et al., "Manipulating microwaves using spatial transformations," in Proc. 4th Int. Topical Meet. Nanophoton. Metamater (NANOMETA). Seefeld, Austria, Jan. 2013. [Online]. Available: http://2013.nanometa.org/files/PreliminaryProgram16November2012.pdf

[29] S. A. R. Horsley, I. R. Hooper, R. C. Mitchell-Thomas, and O. Quevedo-Teruel, "Removing singular refractive indices with sculpted surfaces," Sci. Rep., vol. 4, May 2014, Art. no. 4876. [Online]. Available: http://www.pubmedcentral.nih.gov/articlerender.fcgi?artid=4007085\& tool $=$ pmcentrez\&rendertype $=$ abstract

[30] R. C. Mitchell-Thomas, T. M. McManus, O. Quevedo-Teruel, S. A. R. Horsley, and Y. Hao, "Perfect surface wave cloaks," Phys. Rev Lett, vol. 111, no. 21, Nov. 2013, Art. no. 213901. [Online]. Available: http://journals.aps.org/prl/abstract/10.1103/PhysRevLett.111.213901

[31] T. M. McManus, J. A. Valiente-Kroon, S. A. R. Horsley, and Y. Hao, "Illusions and cloaks for surface waves," Sci. Rep., vol. 4, Aug. 2014, Art. no. 5977. [Online]. Available: http://www.pubmedcentral.nih.gov/ articlerender.fcgi?artid $=4141251 \&$ tool $=$ pmcentrez\&rendertype $=$ abstract

[32] R. C. Mitchell-Thomas, O. Quevedo-Teruel, T. M. McManus, S. A. R. Horsley, and Y. Hao, "Lenses on curved surfaces," Opt. Lett., vol. 39, no. 12, pp. 3551-3554, Jun. 2014. [Online]. Available: http://www.osapublishing.org/viewmedia.cfm?uri=ol-39-12-3551\&seq= $0 \&$ html=true

[33] O. Quevedo-Teruel et al., "Transformation optics for antennas: Why limit the bandwidth with metamaterials?" Sci. Rep., vol. 3, Jan. 2013, Art. no. 1903. [Online]. Available: http://www.nature.com/srep/ 2013/130528/srep01903/full/srep01903.html

[34] C. Mateo-Segura, A. Dyke, H. Dyke, S. Haq, and Y. Hao, "Flat Luneburg lens via transformation optics for directive antenna applications," IEEE Trans. Antennas Propag., vol. 62, no. 4, pp. 1945-1953, Apr. 2014. [Online]. Available: http://ieeexplore.ieee.org/lpdocs/epic03/ wrapper.htm?arnumber $=6719535$

[35] J. C. Maxwell, "Solutions of problems," Cambridge Dublin Math. J., vol. 9, pp. 9-11, Feb. 1854.

[36] R. K. Luneburg, Mathematical Theory of Optics. Berkeley, CA, USA: Univ. California Press, 1964.

[37] Wikipedia. (2017). Luneburg Lens-Wikipedia, The Free Encyclopedia, accessed May 17, 2017. [Online]. Available: https://en.wikipedia. org/w/index.php?title=Luneburg lens\&oldid $=777447331$

[38] U. Leonhardt and T. Philbin, Geometry and Light: The Science of Invisibility. New York, NY, USA: Dover, 2010.

[39] U. Leonhardt and S. Sahebdivan, "Perfect imaging: they do not do it with mirrors," J. Opt., vol. 13, no. 2, Feb. 2011, Art. no. 24016. [Online]. Available: https://doi.org/10.1088/2040-8978/13/2/024016

[40] O. Quevedo-Teruel, R. C. Mitchell-Thomas, and Y. Hao, "Frequency dependence and passive drains in fish-eye lenses," Phys. Rev. A, Gen. Phys., vol. 86, no. 5, Nov. 2012, Art. no. 053817. [Online]. Available: https://dx.doi.org/10.1103/PhysRevA.86.053817

[41] T. Tyc and A. Danner, "Resolution of Maxwell's fisheye with an optimal active drain," New J. Phys., vol. 16, no. 6, Jun. 2014, Art. no. 063001. [Online]. Available: http://stacks.iop.org/1367-2630/16/i=6/a=063001

[42] U. Leonhardt, S. Sahebdivan, A. Kogan, and T. Tyc, "A simple model explaining super-resolution in absolute optical instruments," New J. Phys., vol. 17, no. 5, 2015, Art. no. 053007. [Online]. Available: http://stacks.iop.org/1367-2630/17/i=5/a $=053007$

[43] L. A. Pazynin, V. L. Pazynin, and H. O. Sliusarenko, "Imaging properties of finite Maxwell fish eye medium," IEEE Trans. Antennas Propag., vol. 63, no. 10, pp. 4393-4399, Oct. 2015.

[44] J. C. Garnett and J. G. Valentine, "Maxwell fisheye lens as a waveguide crossing for integrated photonics," in Proc. Conf. Lasers ElectroOpt. (CLEO), May 2012, pp. 1-2.

[45] J. C. Garnett, "Maxwell fisheye lens as a waveguide crossing for integrated photonics," M.S. thesis, Vanderbilt Univ., Nashville, TN, USA, Jun. 2013. [Online]. Available: http://etd.library.vanderbilt.edu/available/ etd-06032013-122649/ 
[46] T. Zentgraf, J. Valentine, N. Tapia, J. Li, and X. Zhang, "An optical 'Janus' device for integrated photonics," Adv. Mater., vol. 22, no. 23 , pp. 2561-2564, Jun. 2010. [Online]. Available: http://dx.doi.org/10. 1002/adma.200904139

[47] H. Kurt, B. B. Oner, M. Turduev, and I. H. Giden, "Modified Maxwell fish-eye approach for efficient coupler design by graded photonic crystals," Opt. Exp., vol. 20, no. 20, pp. 22018-22033, Sep. 2012. [Online]. Available: http://www.opticsexpress.org/abstract.cfm?URI=oe20-20-22018

[48] Y. Shi, K. Li, J. Wang, L. Li, and C.-H. Liang, "An etched planar metasurface half Maxwell fish-eye lens antenna," IEEE Trans. Antennas Propag., vol. 63, no. 8, pp. 3742-3747, Aug. 2015.

[49] H.-X. Xu, G.-M. Wang, Z. Tao, and T. Cai, "An octave-bandwidth half Maxwell fish-eye lens antenna using three-dimensional gradient-index fractal metamaterials," IEEE Trans. Antennas Propag., vol. 62, no. 9, pp. 4823-4828, Sep. 2014.

[50] M. Huang, S. Yang, F. Gao, R. Quarfoth, and D. Sievenpiper, "A 2-D multibeam half Maxwell fish-eye lens antenna using high impedance surfaces," IEEE Antennas Wireless Propag. Lett., vol. 13, pp. 365-368, 2014.

[51] Z. L. Mei, J. Bai, T. M. Niu, and T. J. Cui, "A half Maxwell fish-eye lens antenna based on gradient-index metamaterials," IEEE Trans. Antennas Propag., vol. 60, no. 1, pp. 398-401, Jan. 2012.

[52] B. Fuchs et al., "Comparative design and analysis of luneburg and half Maxwell fish-eye lens antennas," IEEE Trans. Antennas Propag., vol. 56 , no. 9, pp. 3058-3062, Sep. 2008.

[53] B. Fuchs, O. Lafond, S. Rondineau, M. Himdi, and L. L. Coq, "Off-axis performances of half Maxwell fish-eye lens antennas at $77 \mathrm{GHz}$," IEEE Trans. Antennas Propag., vol. 55, no. 2, pp. 479-482, Feb. 2007.

[54] D. M. Pozar, Microwave Engineering, 2nd ed. Hoboken, NJ, USA: Wiley, 1998.

[55] A. M. Nicolson and G. F. Ross, "Measurement of the intrinsic properties of materials by time-domain techniques," IEEE Trans. Instrum. Meas., vol. IM-19, no. 4, pp. 377-382, Nov. 1970.

[56] W. B. Weir, "Automatic measurement of complex dielectric constant and permeability at microwave frequencies," Proc. IEEE, vol. 62, no. 1, pp. 33-36, Jan. 1974.

[57] A. Sihvola, Electromagnetic Mixing Formulas and Applications. Stevenage, U.K.: The Institution of Engineering and Technology, 2008

[58] L. Jylhä and A. Sihvola, "Equation for the effective permittivity of particle-filled composites for material design applications," J. Phys. D, Appl. Phys., vol. 40, no. 16, Aug. 2007, Art. no. 4966. [Online]. Available: http://stacks.iop.org/0022-3727/40/i=16/a=032

[59] Q. Lei, "All-dielectric composites for metamaterial applications," Ph.D. dissertation, Dept. Mater., Univ. Oxford, Oxford, U.K., 2016

[60] T. Yamada, T. Ueda, and T. Kitayama, "Piezoelectricity of a highcontent lead zirconate titanate/polymer composite," J. Appl. Phys., vol. 53, no. 6, pp. 4328-4332, Jun. 1982. [Online]. Available: http://scitation.aip.org/content/aip/journal/jap/53/6/10.1063/1.331211

[61] N. K. James, D. van den Ende, U. Lafont, S. van der Zwaag, and W. A. Groen, "Piezoelectric and mechanical properties of structured PZT-Epoxy composites," J. Mater. Res., vol. 28, no. 4, pp. 1-7, Feb. 2013. [Online]. Available: http://www.journals.cambridge. org/abstract_S0884291412004281
[62] X. Zhao, C. Hinchliffe, C. Johnston, P. J. Dobson, and P. S. Grant, "Spray deposition of polymer nanocomposite films for dielectric applications," Mater. Sci. Eng. B, vol. 151, no. 2, pp. 140-145, Jun. 2008. [Online]. Available: http://www.sciencedirect.com/science/article/ pii/S0921510708002377

[63] Y. Rao, J. Qu, T. Marinis, and C. P. Wong, "A precise numerical prediction of effective dielectric constant for polymer-ceramic composite based on effective-medium theory," IEEE Trans. Compon. Packag. Technol., vol. 23, no. 4, pp. 680-683, Dec. 2000.

[64] S.-D. Cho, S.-Y. Lee, J.-G. Hyun, and K.-W. Paik, "Comparison of theoretical predictions and experimental values of the dielectric constant of epoxy/BaTiO 3 composite embedded capacitor films," J. Mater. Sci., Mater. Electron., vol. 16, no. 2, pp. 77-84, Feb. 2005. [Online]. Available: http://dx.doi.org/10.1007/s10854-005-6454-3

[65] P. Thomas, K. Varughese, K. Dwarakanath, and K. Varma, "Dielectric properties of poly(vinylidene fluoride) $/ \mathrm{CaCu}_{3} \mathrm{Ti}_{4} \mathrm{O}_{1} 2$ composites," Comp. Sci. Technol., vol. 70, no. 3, pp. 539-545, Mar. 2010 [Online]. Available: http://www.sciencedirect.com/science/article/pii/ S0266353809004485

[66] Y.-L. Su, C. Sun, W.-Q. Zhang, and H. Huang, "Fabrication and dielectric properties of $\mathrm{Na}_{0} .5 \mathrm{Bi}_{0} .5 \mathrm{Cu}_{3} \mathrm{Ti}_{4} \mathrm{O}_{1} 2 /$ poly (vinylidene fluoride) composites," Mater. Sci., vol. 48, no. 23, pp. 8147-8152, Dec. 2013. [Online]. Available: http://dx.doi.org/10.1007/s10853-013-7627-8

[67] M. P. McNeal, S.-J. Jang, and R. E. Newnham, "The effect of grain and particle size on the microwave properties of barium titanate $\left(\mathrm{BaTiO}_{3}\right)$," J. Appl. Phys., vol. 83, no. 6, pp. 3288-3297, Jun. 1998. [Online]. Available: http://dx.doi.org/10.1063/1.367097

[68] K.-C. Cheng, C.-M. Lin, S.-F. Wang, S.-T. Lin, and C.-F. Yang, "Dielectric properties of epoxy resin-barium titanate composites at high frequency," Mater. Lett., vol. 61, no. 3, pp. 757-760, 2007. [Online]. Available: http://www.sciencedirect.com/science/article/pii/ S0167577X06006641

[69] D. Isakov, C. J. Stevens, F. Castles, and P. S. Grant, "3D-printed high dielectric contrast gradient index flat lens for a directive antenna with reduced dimensions," Adv. Mater. Technol., vol. 1, no. 6, p. 1600072, Sep. 2016. [Online]. Available: http://dx.doi.org/10.1002/admt. 201600072

[70] D. Isakov, C. J. Stevens, F. Castles, and P. S. Grant, "A split ring resonator dielectric probe for near-field dielectric imaging," Sci. Rep., vol. 7, p. 2038, May 2017.

Qin Lei, photograph and biography not available at the time of publication.

Robert Foster (M'08), photograph and biography not available at the time of publication.

Patrick S. Grant, photograph and biography not available at the time of publication.

Chris Grovenor, photograph and biography not available at the time of publication 